\title{
The Correlation between Femoral Anteversion and Femoral Condyle Rotation in Patients with Osteoarthritis of the Hip Joint: Investigation about the Asian Female Patients
}

\author{
Hiroshi Yonezu*, Hiroshi Mikami, Koichi Oba, Katsutoshi Miyatake, \\ Michihiro Takai, Akihiro Nitta \\ Department of Orthopedics, Yoshinogawa Medical Center, Tokushima, Japan \\ Email: ^hyonezu@kj9.so-net.ne.jp
}

How to cite this paper: Yonezu, H., Mikami, H., Oba, K., Miyatake, K., Takai, M. and Nitta, A. (2017) The Correlation between Femoral Anteversion and Femoral Condyle Rotation in Patients with Osteoarthritis of the Hip Joint: Investigation about the Asian Female Patients. Open Journal of Orthopedics, 7, 369-374. https://doi.org/10.4236/ojo.2017.711038

Received: October 12, 2017

Accepted: November 11, 2017

Published: November 14, 2017

Copyright $\odot 2017$ by authors and Scientific Research Publishing Inc. This work is licensed under the Creative Commons Attribution International License (CC BY 4.0).

http://creativecommons.org/licenses/by/4.0/

\begin{abstract}
An abnormality of femoral anteversion (FA) is often recognized in patients with osteoarthritis of the hip joint (hip OA). And it is considered that rotation abnormality in the knee and the lower leg occurs as compensation, in cases of FA abnormality. The purpose of this study was to assess the magnitude and variability of FA and femoral condyle rotation (FCR) in order to identify the association between FA and FCR in female patients with hip OA who require total hip arthroplasty. A total of 174 hips from 174 hip OA patients undergoing THA were included in this study. All patients were Asian women (average age: 71.1 years). An equilateral correlation was accepted in FA and FCR. It was thought that FA abnormality might be accompanied by morphological rotation abnormalities of the knee joint. Caution is needed in considering femoral anteversion using the posterior condylar line. We must consider FA in patients undergoing THA after having considered the whole leg rotation.
\end{abstract}

\section{Keywords}

Femoral Anteversion, Femoral Condyle Rotation, Osteoarthritis of the Hip, Posterior Condylar Line, Epicondylar Line, Total Hip Arthroplasty (THA)

\section{Introduction}

An abnormality of femoral anteversion (FA) is often recognized in patients with osteoarthritis of the hip joint (hip OA). Sugano et al. [1] reported that FA of developmental dysplasia of the hip had more anteversion than the control group. An abnormality of FA may affect the pathogenesis of hip OA [2]. FA describes 
the rotation of the femur between the proximal end (femoral neck) and the distal end (femoral condyle). FA is usually measured as the angle between the posterior condylar line (PC line) and the femoral neck, using computed tomography (CT). On the other hand, it is recommended that the femoral component should be inserted parallel to the epicondylar line (EP line) when total knee arthroplasty (TKA) is performed [3] [4]. In other words, we assume that the PC line is the base line when we perform total hip arthroplasty (THA), and assume that the EP line is the base line when we perform TKA. It is considered that rotation abnormality in the knee and the lower leg occurs as compensation, in cases of FA abnormality. Thus, it can be argued that, there is a variation of the femoral condyle rotation (FCR), in these scenarios that is, in part, becoming a reference for the FA, itself. However, there are few reports on the relationship between FA and FCR. The purpose of this study was to assess the magnitude and variability of FA and FCR in order to identify the association between FA and FCR in female patients with hip OA who require THA.

\section{Patients and Methods}

A total of 174 hips from 174 hip OA patients undergoing THA in Yoshinogawa Medical Center between January 2013 and July 2017 were included in this study. All patients were Asian women (average age: 71.1 years). Individuals with past illnesses that influenced the hip and knee joints, those with a history of leg operation, and those that did not have correctly recorded CT scans were not included.

We measured FA and FCR using multi-planar reconstruction images obtained from the CT. The anteversion of the femur was defined according to Kingsley et al. [5], while the axis of the femoral shaft was defined according to Sugano et al. [6] A definition of FCR designated it to be an angle comprised by the intersection of the EP line and the PC line (Figure 1, Figure 2).

For an index of acetabular dysplasia, acetabular angle was defined according to Sharp [7] with a hip radiograph image (Figure 3). The authors obtained consent in doing CT photography or radiography from the patient.

Values are shown as mean \pm standard error (SE). Correlations between two independent measurements were assessed using Pearson's correlation coefficient. Differences were considered statistically significant for $\mathrm{p}$ values $<0.05$. All

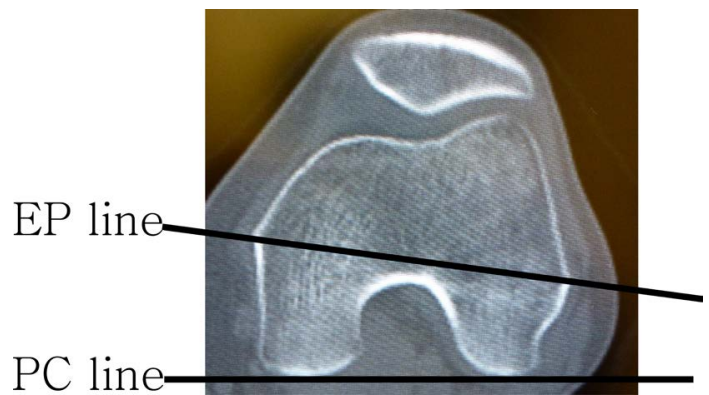

Figure 1. Posterior condylar line (PC line) and epicondylar line (EP line). 


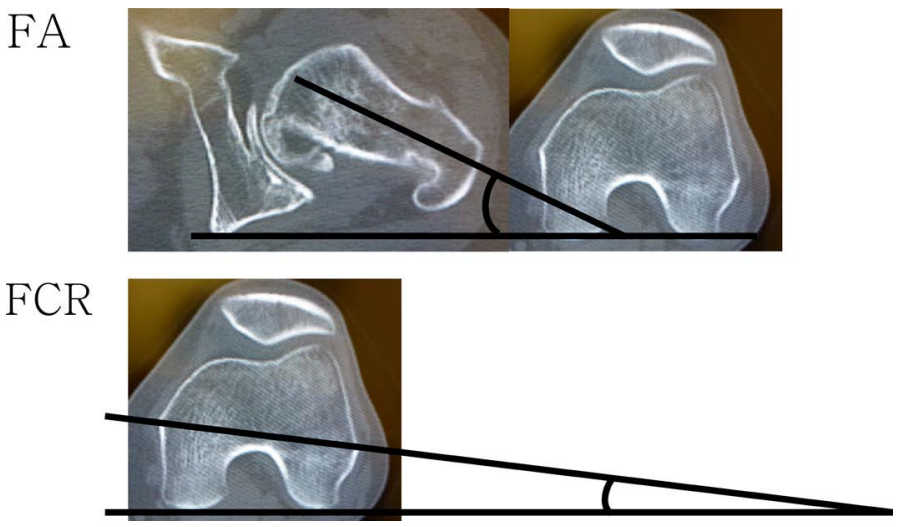

Figure 2. Measurement of femoral anteversion (FA) and femoral condyle rotation (FCR).

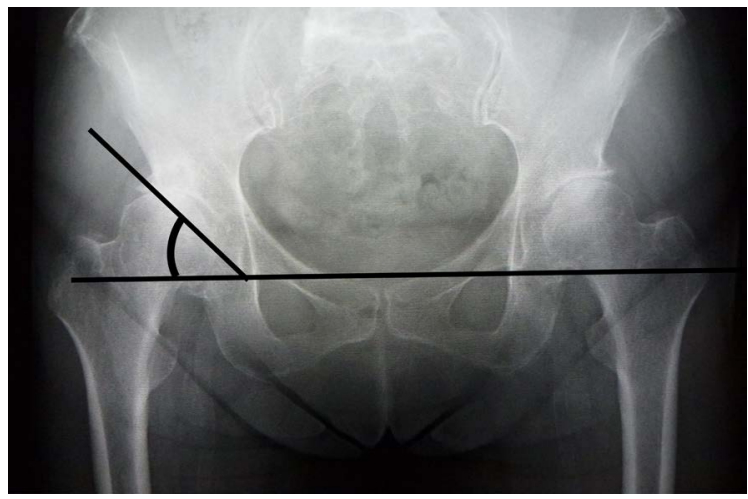

Figure 3. Measurement of acetabular angle.

statistical analyses were performed using SPSS version 21.0 (IBM Corp., Armonk, NY).

\section{Results}

The average FA value of the subjects was $22.9^{\circ} \pm 13.1^{\circ}$ (range: $-3.5^{\circ}-69.5^{\circ}$ ) FCR value was $6.4^{\circ} \pm 2.4^{\circ}$ (range: $-5.0^{\circ}-12.0^{\circ}$ ). The acetabular angle was $42.1^{\circ}$ $\pm 5.1^{\circ}$ (range: $30.7^{\circ}-61.0^{\circ}$ ). An equilateral correlation was accepted in FA and FCR. $(\mathrm{r}=0.467, \mathrm{p}<0.01)$ (Figure 4$)$ As acetabular angle increased, the FA tended to increase. However, the significant correlation was not accepted in acetabular angle and FA (Figure 5).

\section{Discussion}

FA abnormality must be considered in patients undergoing THA because of possible impingement and dislocation. In late years, an implant of the modular neck type is often used for correcting FA abnormality. Modular neck is theoretically superior; however, several studies have reported no distinctive change in the dislocation rate, compared to conventional THA [8] [9] [10] [11]. These reports show that certain leg movements, such as rotation, in the activities of daily living(ADL) are influenced by not only the rotation of the hip joint but also by rotation of the knee joint and lower leg. However, few reports have examined 


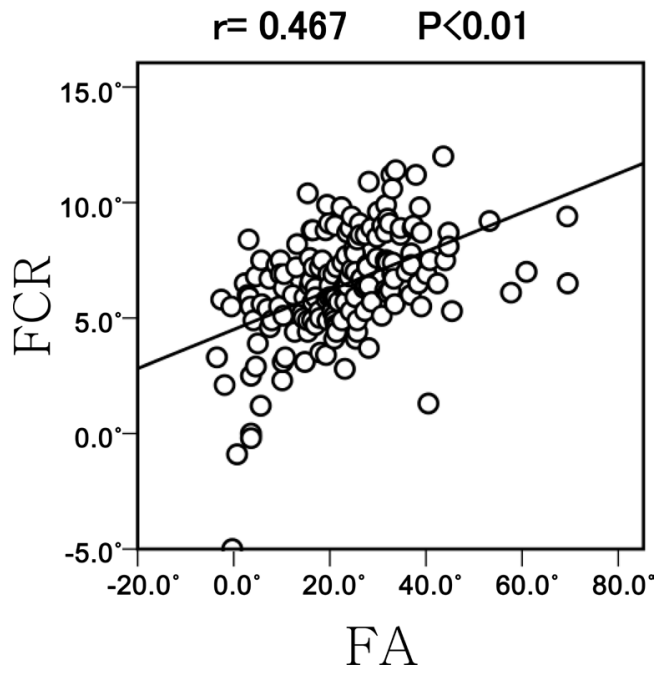

Figure 4. Correlation between femoral anteversion (FA) and femoral condyle rotation (FCR).

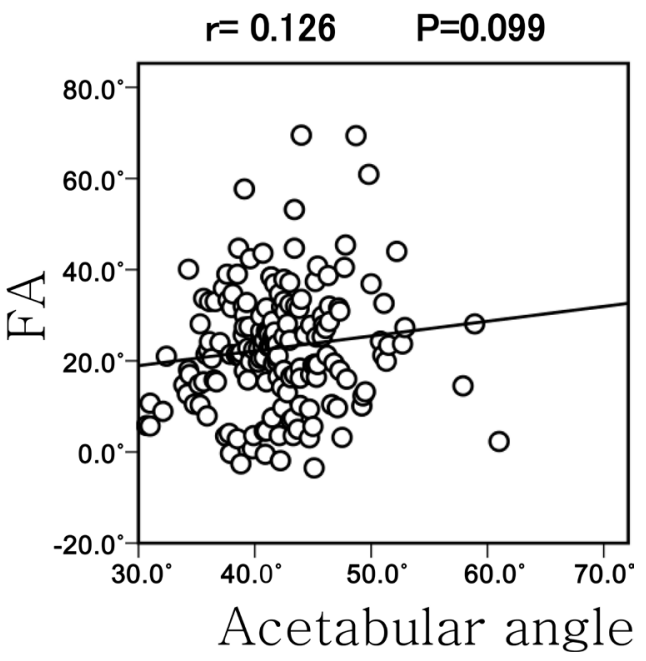

Figure 5. Correlation between acetabular angle and femoral anteversion (FA).

that whether there is a change in functional leg rotation after THA. Uemura $\mathrm{K}$ et al. [12] reported individual variance and sex difference in the position of the PC line measured before and after THA, and suggested that caution may be needed when using it as a rotational reference. Alternatively, compensation for rotation that occurs at the knee is reported in cases of FA abnormality. A positive correlation between femoral rotation and tibial torsion (TT) using in vitro measurement of anatomic specimens was reported by Kobyliansky E et al. [13] Additionally, Rittmeister $M$ et al. [14] reported that there was an equilateral association between FA and TT in patients with hip OA, and reported that they may produce rotational imbalance of leg and postoperative gait disturbance if they were to correct FA by an operation in defiance of TT. In this study, FA had an equilateral association with FCR, and it was thought that FA abnormality might be accompanied by morphological rotation abnormalities of the knee joint.

It is necessary to assess the association between functional rotation of leg in 
ADL movements and the morphological rotation of the hip and knee joint, via motion analysis or other methods. In the future, we must consider the abnormality of FA in patients undergoing THA after having considered the whole leg rotation.

There are several limitations to this study.

First, the subjects of this study were advanced or terminal hip OA patients undergoing THA. Patients with normal hips or those in an early stage of hip OA were not evaluated. It is difficult to obtain consent in doing CT photography from these patients. Second, we were unable to consider sex difference, because there were few male patients in this investigation. It is well known that there are sex differences as for muscle strength and muscle balance. And these differences may be responsible for the sex difference as for FA. Third, we were unable to consider the influence of acetabular version. Morphological correlation between the acetabulum version and FA of the hip joint is controversial. Akiyama et al. [15] reported that FA is correlated with acetabular version and coverage in Asian women. Alternatively, Mootha et al. [16] reported that there was no correlation between FA and acetabular version. Thus, future studies should be carried out to evaluate these problems.

\section{Conclusion}

FA was significantly correlated with FCR in Asian female patients with osteoarthritis of the hip joint. Caution is needed in considering femoral anteversion using the PC line. We must consider FA in patients undergoing THA after having considered the whole leg rotation.

\section{Declaration of Conflicting Interests}

The authors declare that there is no conflict of interest regarding the publication of this paper.

\section{References}

[1] Sugano, N., Noble, P.C., Kamaric, E., Salma, J.K., Ochi, T. and Tullos, H.S. (1998) The Morphology of the Femur in Developmental Dysplasia of the Hip. Journal of Bone and Joint Surgery British Volume, 80, 711-719. https://doi.org/10.1302/0301-620X.80B4.8319

[2] Zeng, W.N., Wang, F.Y., Chen, C., Zhang, Y., Gong, X.Y., Zhou, K., Chen, Z., Wang, D., Zhou, Z.K. and Yang, L. (2016) Investigation of Association between Hip Morphology and Prevalence of Osteoarthritis. Scientific Reports, 6, 23477. https://doi.org/10.1038/srep23477

[3] Berger, R.A., Rubash, H.E., Seel, M.J., Thompson, W.H. and Crossett, L.S. (1993) Determining the Rotational Alignment of the Femoral Component in Total Knee Arthroplasty Using the Epicondylar Axis. Clinical Orthopaedics and Related Research, 286, 40-47.

[4] Matsuda, S., Miura, H., Nagamine, R., Mawatari, T., Tokunaga, M., Nabeyama, R. and Iwamoto, Y. (2004) Anatomical Analysis of the Femoral Condyle in Normal and Osteoarthritic Knees. Journal of Orthopaedic Research, 22, 104-109. 
https://doi.org/10.1016/S0736-0266(03)00134-7

[5] Kingsley, P.C., Olmsted, K.L. and Michigan, A.A. (1948) A Study to Determine the Angle of Anteversion the Neck of the Femur. Journal of Bone and Joint Surgery American Volume, 30, 745-751. https://doi.org/10.2106/00004623-194830030-00021

[6] Sugano, N., Noble, P.C. and Kamaric, E. (1998) A Comparison of Alternative Methods of Measuring Femoral Anteversion. Journal of Computer Assisted Tomography, 22, 610-614. https://doi.org/10.1097/00004728-199807000-00019

[7] Sharp, I.K. (1961) Acetabular Dysplasia: The Acetabular Angle. Journal of Bone and Joint Surgery British Volume, 43, 268-272.

[8] Duwelius, P.J., Burkhart, B., Carnahan, C., Branam, G., Ko, L.M., Wu, Y., Froemke, C., Wang, L. and Grunkemeiser, G. (2014) Modular versus Nonmodular Neck Femoral Implants in Primary Total Hip Arthroplasty: Which Is Better? Clinical Orthopaedics and Related Research, 472, 1240-1245.

https://doi.org/10.1007/s11999-013-3361-4

[9] Fitch, D.A., Ancarani, C. and Bordini, B. (2015) Long-Term Survivorship and Complication Rate Comparison of a Cementless Modular Stem and Cementless Fixed Neck Stems for Primary Total Hip Replacement. International Orthopaedics, 39, 1827-1832. https://doi.org/10.1007/s00264-015-2894-4

[10] Gerhardt, D.M., Bisseling, P., de Visser, E. and van Susante, J.L. (2014) Modular Necks in Primary Hip Arthroplasty without Anatomical Deformity: No Clear Benefit on Restoration of Hip Geometry and Dislocation Rate. An Exploratory Study. Journal of Arthroplasty, 29, 1553-1558. https://doi.org/10.1016/j.arth.2014.02.009

[11] Gofton, W.T., IIIical, E.M., Feibel, R.J., Kim, P.R. and Beaule, P.E. (2017) A Single-Center Experience with a Titanium Modular Neck Total Hip Arthroplasty. Journal of Arthroplasty, 32, 2450-2456. https://doi.org/10.1016/j.arth.2017.03.025

[12] Uemura, K., Takao, M., Sakai, T., Nishii, T. and Sugano, N. (2016) The Validity of Using the Posterior Condylar Line as a Rotational Reference for the Femur. Journal of Arthroplasty, 31, 302-306. https://doi.org/10.1016/j.arth.2015.08.038

[13] Kobyliansky, E., Weissman, S.L. and Nathan, H. (1979) Femoral and Tibial Torsion: A Correlation Study in Dry Bones. International Orthopaedics, 3,145-147.

[14] Rittmeister, M., Hanusek, S. and Starker, M. (2006) Does Tibial Rotation Correlate with Femoral Anteversion? Implications for Hip Arthroplasty. Journal of Arthroplasty, 21, 553-558. https://doi.org/10.1016/j.arth.2005.05.032

[15] Akiyama, M., Nakashima, Y., Fujii, M., Sato, T., Yamamoto, T., Mawatari, T., Motomura, G., Matsuda, S. and Iwamoto, Y. (2012) Femoral Anteversion Is Correlated with Acetabular Version and Coverage in Asian Women with Anterior and Global Deficient Subgroup of Hip Dysplasia: A CT Study. Skeletal Radiology, 41, 1411-1418. https://doi.org/10.1007/s00256-012-1368-7

[16] Mootha, A.K., Saini, R., Dhillon, M.S., Aggarwal, S., Kumar, V. and Tripathy, S.K. (2010) MRI Evaluation of Femoral and Acetabular Anteversion in Developmental Dysplasia of the Hip. A Study in an Early Walking Age Group. Acta Orthopaedica Belgica, 76, 174-180. 